\title{
Application Research of Non-financial Indicators in the Evaluation of Enterprise Performance
}

\author{
Gang Chen ${ }^{1,2^{*}}$, Wendong Zhao ${ }^{2}$
}

\author{
${ }^{I}$ North Jiangsu Development Institute, Jiangsu, China \\ ${ }^{2}$ Huaiyin Institute of Technology, Huaiyin, China \\ *Corresponding author. Email: cg1972@hyit.edu.cn
}

\begin{abstract}
To combine financial indicators with non-financial indicators to evaluate corporate performance is increasingly valued by the theoretical and practical circles. It is generally believed that the inclusion of nonfinancial indicators in the evaluation system can make up for the shortcomings and deficiencies of financial indicators, thus making performance evaluation more scientific and reasonable, and the scope of application more extensive. This paper studies the motivation of the reform of corporate performance evaluation mechanism, analyzes the advantages and disadvantages of non-financial indicators, and reveals the four principles of non-financial indicators selection: strategic principles, key principles, weak collinearity principles, strong consistency principles. Finally, it puts forward the non-financial indicator measures to improve the enterprise performance evaluation system: increasing the enterprise's comprehensive competitiveness indicator, growth performance indicator and cultural performance indicator. This research enriches the literature on the use of non-financial indicators in China's performance evaluation system, and also contributes to the overall comprehensive performance evaluation of Chinese enterprises, thereby improving the operational efficiency and development potential of enterprises.
\end{abstract}

Keywords: Performance evaluation, non-financial indicators, financial indicators, strategic principles

\section{INTRODUCTION}

Non-financial indicators are relative to financial indicators. They can effectively compensate for the deficiencies and defects of traditional financial indicators in evaluating corporate performance, and are an important part of the enterprise performance evaluation system. The financial indicators mainly reflect the production and operation status of the enterprise through the financial data provided by the financial management activities of the enterprise, such as the efficiency of capital use, the solvency of the enterprise, the efficiency of enterprise asset management, the profitability of the enterprise, etc. Non-financial indicators can't be analyzed and described simply by the financial data of the company, such as the company's sustainable development ability, the company's green production capacity, the company's social responsibility capacity, and customer satisfaction. Non-financial indicators are obtained through a series of data collection and processing and some special analysis methods. Therefore, the use of financial indicators to measure is in conjunction with the use non-financial indicators for description and analysis, which is of great significance to accurately reflect the performance of production and operation of enterprises and to motivate business managers. Non-financial measurement and evaluation is also an indispensable part of the corporate performance evaluation system.

\section{THE ANALYSIS OF THE REFORM MOTIVATION OF ENTERPRISE PERFORMANCE EVALUATION MECHANISM}

\subsection{Limitations of Financial Indicators}

The traditional enterprise performance evaluation system is based on financial indicators and data. It measures corporate performance only through some financial indicators and data such as asset-liability ratio, profit rate, capital turnover rate, earnings per share, and return on assets. Although these indicators can also reflect the performance of enterprises, they have a common shortcoming, that is, they reflect the past performance of the company, but they cannot predict the future development of the company, which may lead to the damage of long-term interests caused by the pursuit of short-term interests. Non-financial indicators such as organizational innovation, corporate social identity, and customer satisfaction are more representative of the potential and motivation of the company's future development. Moreover, non-financial indicators are not easy to be manipulated and fraudulent, and are more realistic and easier to understand. In addition, nonfinancial indicators can also make up for the shortcomings 
of financial indicators that can only be quantified, and scientifically reflect the intangible assets of enterprises. Therefore, many companies are trying to build a more complete and scientific evaluation system by introducing non-financial indicators to more fully reflect corporate performance.

\subsection{The Tremendous Pressure Brought About by Changes in the External Environment of The Company}

With the development and progress of the times, especially since the advent of economic globalization since the 21st century, the survival and business environment of enterprises have undergone tremendous changes. In the context of information age, the focus of competition between enterprises has gone from the management of tangible assets to the development and utilization of intangible assets. The increasingly important role of nonfinancial value drivers in corporate competition requires that business managers look for new non-financial indicators to measure them. Companies that still focus on the numbers provided by financial accounting are difficult to stand in the future.

\subsection{The Traditional Performance Evaluation System cannot Meet the Increasing Demand of Management Innovation for Information}

In order to gain an advantage in market competition, Companies are constantly innovating in management. Management innovation requires a large amount of information support, including financial information, production information, business information and other aspects of information, which are difficult to provide in the traditional performance evaluation system. Therefore, it is necessary to build a new enterprise performance evaluation system to provide more information support for management innovation.

\section{ANALYSIS OF THE ADVANTAGES AND DISADVANTAGES OF NON- FINANCIAL INDICATORS}

\subsection{Analysis of the Advantages of Non- Financial Indicators}

Financial indicators do not reflect the future value that companies can create, but only the past performance of companies. In contrast, non-financial indicators more reflect future information, including improving customer satisfaction and maintaining the credibility of the company among customers, gaining market share by establishing markets, developing new products, and opening up market sales channels. By gradually improving these indicators, companies can gradually improve their original business performance and make a leap forward.

In general, financial indicators do not reflect the various factors that affect the performance of an enterprise, especially those factors that affect business performance but are difficult to measure. There is serious information asymmetry in china's current financial reporting system and accounting information system and development is relatively late. If only financial indicators are used to evaluate enterprise accounting information, once the accounting information has some unexpected abnormal conditions, such as data loss, major changes and turbulence in the external environment, the evaluation results cannot objectively and truly reflect the actual situation, making financial information collectors face difficulties. The introduction of non-financial indicators can revise and supplement the results of financial indicators evaluation from the external environment from a non-financial perspective.

There are many advantages to establish non-financial indicators evaluation system. Through comprehensive evaluation of corporate performance, it prevents and eliminates certain short-sighted behaviors, prompts enterprises to make comprehensive development strategies from a long-term perspective, promotes the formation of self-motivation and control mechanisms, and promote the integration of the company's own management and operation methods with the market economy. Nonfinancial indicators can be used in combination with financial indicators to comprehensively, synthetically and systematically evaluate corporate performance, and can be used separately to qualitatively evaluate firm performance. This is possible for certain companies that lack basic accounting information, data failures, or do not need to do quantitative analysis in the evaluation process.

\subsection{Analysis of the Shortcomings of Non- financial Indicators}

Non-financial indicators are important, but in practice, there are still some shortcomings in non-financial indicators. In general, there are mainly the following deficiencies and shortcomings:

\subsubsection{Analysis of the difficulty and cost of collecting non-financial indicators}

For example, the non-financial indicators involved in the Balanced Scorecard have three aspects: external customers, internal processes, and innovative learning. Taken together, these three aspects have about 30 indicators each. Although different companies apply different indicators, in general, the indicator system is extremely large. In addition, most of these indicators are information that is not easy to accurately collect. The collection of data relies on experimental research methods, 
such as questionnaires, and the difficulty is obvious. At the same time, due to the liquidity of customers and the limitations of survey technology, the cost of data collection will be relatively large.

\subsubsection{Since non-financial indicators are applied to the value creation level of the production and operation process, changes in non-financial indicators always lag behind financial performance}

This shortcoming may lead to problems in the use of nonfinancial indicators. For example, management may therefore consider non-financial indicators to be slow to refuse to adopt this performance assessment method, or can't choose the best non-financial evaluation indicators.

\subsubsection{Many of the non-financial indicators are not easily quantifiable}

Such as customer loyalty, and brand recognition. cannot be converted into exact quantitative indicators. Because the indicators that are not quantifiable are not objective enough, the employees will have the feeling that the fate is controlled by the supervisor, which makes the employees more to please the supervisors, and it is not easy to motivate the employees to serve the customers and implement the company strategy.

\subsubsection{The number of non-financial indicators is difficult to grasp}

Non-financial indicators are not as good as possible, and their quantity and category should play an active role in an appropriate scope. If the pursuit of quantity increase is not achieved, it will not only achieve the prior effect, if simply pursue the increase in quantity, it will not achieve the preeffect, but will affect the normal operation and implementation of the entire evaluation system. So choosing the right non-financial indicators is an important job. At the same time, there may be situations of mutual exclusion and contradiction between certain indicators, so conflicts between departments are also inevitable.

\section{NON-FINANCIAL INDICATOR SELECTION PRINCIPLE}

With the popularity of the Balanced Scorecard, many companies have begun to cite non-financial indicators, but because there is no scientific way to determine the nonfinancial indicators that are suitable for the business and really have an impact when selecting indicators, Most of them choose to apply and imitate some non-financial performance evaluation frameworks, which are easy to produce some even serious mistakes. Therefore, when selecting non-financial indicators, the following two factors need to be considered: first, the representativeness of individual indicators; second, the internal structure of the indicator system. The representation of non-financial indicators mainly refers to the strategic orientation and key of the indicators, while the internal structure of the indicator system mainly shows the strong consistency of the evaluation objectives and the weak collinearity of the evaluation contents between the non-financial indicators and the financial indicators.

\subsection{Strategic Principles Drive and Spur the Growth and Improvement of Strategic Factors that are Conducive to Corporate Development}

Strategy is the starting point of the performance evaluation indicator system. Strategic management theory believes that non-financial indicators are used to compensate for the short-term orientation of financial performance indicators, allowing companies to focus on the key factors driving the long-term development of enterprises. A good non-financial indicator needs to fully play its role in organizational performance control and guidance. When selecting non-financial indicators, enterprises should carefully consider the degree of control of indicators on key aspects of the organization and the guiding role of organizational development. It is the strategy of the company that decides which aspects of the organization are key and which promotes development. Therefore, the first principle for companies to choose non-financial indicators is the strategic principle that drives and spurs the growth and improvement of those strategic factors that are conducive to corporate development.

Research shows that strategy is the bridge from nonfinancial performance to financial performance. The strategy guides the direction of non-financial indicators and verifies the effectiveness of the indicators by transforming them into financial performance and contributing to the strategic objectives. Therefore, following the strategic principle, we can avoid the misunderstanding of the weak correlation between nonfinancial performance indicators and financial performance.

\subsection{Key Principle Needs to Fully Represent the Key Links that Affect Organizational Performance in Order to Contribute to Financial Results}

The choice of non-financial indicators is subject to the principle of simplicity, and the use of a small number of performance indicators to measure the real performance of the organization is the way to performance management. Non-financial indicators need to adequately represent the key aspects that affect organizational performance in order 
to contribute to financial results. Otherwise, the cost of using non-financial indicators may be much greater than the return on organizational profits.

Because the impact of non-financial indicators on financial performance results is implicit, the current measurement of non-financial performance is also relatively rough. Key non-financial performance indicators are often determined by managers' imaginations, lacking data and theoretical support. How to choose key non-financial indicators must follow the following two principles: (1) Vertical positive correlation principle. One way to select key non-financial indicators is to longitudinally sort through the enterprise value chain and select indicators that are important to promote financial performance based on positive correlation with financial results. (2) The principle of horizontal anti-correlation. Another approach is to select indicators that have a significant impact on financial performance based on the key links in the enterprise value chain and based on the inverse correlation with financial results.

\subsection{Weak Collinearity Means that there is no Double Counting of Performance Content When Measuring Organizational Performance}

An important principle for evaluating the quality of the indicator system is the weak collinearity between the indicators. Weak collinearity is especially important for the performance evaluation indicator system of enterprises. The collinearity of performance evaluation indicators means the repeated calculation of performance content, which leads to the failure to truly reflect the business performance of the company when measuring organizational performance, and a phenomenon of false high or low; When measuring individual performance, it is impossible to truly reflect the employee's contribution to profit, and the performance is exaggerated or reduced. In the worst case, if the organizational performance is too high and the individual performance is exaggerated, then the enterprise performance loophole will cause huge losses to the enterprise.

In practice, the performance of some companies is very good, but the profits have not risen; some companies have poor organizational performance, but the profits have not been damaged. The enterprise research value chain, trying to find out the links that lead to the increase and decrease of corporate profits. In fact, the collinearity problem of the performance indicator system is not solved, finding the link of increasing or decreasing profits cannot overcome the disconnection contradiction between the results of performance evaluation and corporate profits. There are also companies that have learned the experience in multiple performance evaluation feedbacks, and what can be done to achieve twice the result with half the effort. The collinearity of performance indicators guides employees to tend to achieve the best performance in one aspect to achieve excellence. As a result, some companies have begun to study the weight setting of performance indicators, and want to balance the indicators by weight, but it only dilutes the key performance but does not reduce the staff's performance collapse.

The choice of non-financial indicators should consider the weak collinearity between non-financial indicators and the weak collinearity between non-financial and financial indicators. Non-financial indicators with strong collinearity with financial indicators will lead to repeated calculation of performance, and fail to play the role of non-financial indicators in monitoring and supplementation of financial indicators and profit formation process, thus increasing the internal friction and failing to achieve the purpose of continuously increasing corporate financial performance.

\subsection{Strong Consistency Ensures that the Goals of Non-financial Indicators are Consistent with the Corporate Strategic Orientation, the Assessment Content, Financial Indicators}

The principle of strong consistency refers to ensuring that when setting non-financial indicators, the objectives of non-financial indicators are consistent with the strategic orientation of enterprises, the contents to be assessed and the objectives of financial indicators. The first two consistency are the ones that must be followed when designing performance indicators, and consistent with the goal of financial indicators is easy to be ignored by many enterprises.

Most companies' understanding of non-financial indicators is prone to two mistakes. First, it is easy to completely align non-financial indicators with financial indicators. For example, short-term and long-term, past and future, onesided and comprehensive, think the purpose of the two performance indicators is different. This kind of mistake ignores the causal link between non-financial indicators and financial performance. The performance of all activities of the enterprise is ultimately reflected in financial performance. Non-financial performance ultimately needs to be transformed into financial performance to realize its own value. Therefore, in a sense, non-financial performance is an untransformed, implicit financial performance. For example, the principal-agent theory holds that the purpose of non-financial indicators is to control the behavior of managers to ensure the interests of shareholders; the stakeholder theory holds that nonfinancial indicators are used to balance the interests of all parties in the business environment. Whether it is the interests of shareholders or other stakeholders, the focus of contradiction is the distribution of interests, and the root cause is the distribution of profits. Therefore, in essence, the objectives of non-financial performance indicators should be consistent with the objectives of the financial performance indicators.

The second mistake is to use experience and intuition to set the non-financial indicators that may be effective without verifying the causal link between non-financial indicators and financial performance. This error ignores 
the negative effects of non-financial indicators. Common non-financial indicators are mainly divided into: (1) business process indicators; (2) interest-related relationship indicators; (3) growth and innovation indicators. Strictly verifying the causal relationship between non-financial indicators and financial performance can ensure the consistency between the nonfinancial indicators selected by the company and the financial indicators.

When introducing non-financial indicators, just because the indicators are considered benign, these non-financial indicators that can promote financial performance are introduced, and there is no harm, and the result is only an increase in the workload of performance appraisal. Due to the difficulty in collecting data of non-financial indicators, and the reliability of data sources is difficult to guarantee, the cost of quantitative assessment is relatively large. Different types of non-financial indicators of different strategies, different industries and different stages of life cycle contribute differently to financial performance, and the costs relative to profit contribution are of course different. When the cost of certain indicators is greater than the profit they contribute, it has a huge negative impact.

\section{PERFECTING COUNTERMEASURES FOR ENTERPRISE PERFORMANCE EVALUATION SYSTEM}

\subsection{Improve the Comprehensive Competitiveness of Enterprises}

Both financial and non-financial indicators should be included in a reasonable performance evaluation indicator system. Non-financial indicators generally include four levels of indicator design, namely employee level, customer level, scientific innovation level and business level. The comprehensive competitiveness of an enterprise can be reflected through the integration and synergy of these four indicators. The following is a detailed explanation of the level of technological innovation and customer.

a) The level of technological innovation. Technological innovation emphasizes the high-tech injection function in the production process. By investing a large amount of money, companies are expected to achieve huge market repercussions and benefits with little time cost. The evaluation indicator design on the level of scientific and technological innovation can include three indicators: $R \& D$ expense rate, which reflects the $R \& D$ cost and utilization of new products; new product R\&D speed, which reflects the efficiency of $R \& D$ of new products. The greater the indicator, the more efficient; new product sales rate and return rate, which reflect the profit potential of the new product being developed.

b) Customer level. Enterprises continue to develop and expand the relationship with customers, and actively explore new customer groups, which can help enterprises achieve their business strategy goals faster and enhance their strength. The performance evaluation indicator system on the customer-level can include three aspects: customer maintenance, customer acquisition rate and product delivery timelines, which are used to indicate customer satisfaction. The higher the indicator value, the better the satisfaction; the market (relative) occupancy rate, which is used to indicate the market share of the product or the position and competitiveness of the company in the market; the profit margin of the customer, which is used to indicate the profit the company receives, and also reflects the gain and consumption of the enterprise in meeting the needs of the consumer, is the embodiment of corporate financial goals.

\subsection{Increase Growth Performance Indicators of Corporate}

Due to the increasingly fierce market competition, the foundation of enterprise survival and development is based on maintaining the core competitive advantage of the company from a strategic perspective to achieve long-term goals. The formation and maintenance of core competitive advantages is determined by the development of many factors. The important factors affecting the strategic business success of the enterprise should be fully reflected in the system of performance evaluation indicators. Obviously, the development of the enterprise performance evaluation indicator system should include growth indicators, such as employee loyalty to the company, cooperation between employees and units, productivity of employees and teams, and performance of employee education and training. Although these indicators are not financial performance indicators, they will have an invaluable key impact on the growth of the company. A growing team shows the incomparable combat power. For the implementation of the company's strategy, it will be in a state of competence and cooperation. It has a critical impact on the overall growth of corporate performance. The growth indicators truly reflect the fundamental differences between the company and its competitors. Excellent companies tend to pay more attention to the setting and extraction of such indicators. In practice, the growth indicator refers to the ability to complete the outcome indicators, including three aspects, namely knowledge, skills and willingness. The setting of growth indicators should follow the principle of gradual and orderly progress according to the stage of enterprise development, the ability difference of undertaking objects (departments, individuals), and set up two or three indicators at a time, and adjust dynamically in stages. The establishment of growth performance indicators can not only consider the growth needs of departments and enterprises as a whole, but also take into account the achievement of outcome indicators. 


\subsection{Increase Corporate Cultural Performance Indicators}

In addition to figures of financial revenue and profitability, corporate performance is also reflected in the culture that cannot be shown by financial figures. For example, the corporate cultural value of the company is affirmed by all the employees, which is not shown in the financial figures, but these cultural performances will eventually produce multiplication effect on the financial performance. Zeng Deming and Chen Liyong (2003) believe that the cultural performance of an enterprise is the sum of the ideas formed in the process of production, operation and management, as well as the embodiment of management thoughts, values and enterprise spirit. Corporate culture performance indicators include material cultural indicators, institutional cultural indicators, spiritual culture and other indicators, such as work environment satisfaction, management system satisfaction, human resources policy recognition, corporate culture satisfaction, business philosophy recognition and other indicators. All enterprises have their own corporate culture, and corporate culture has a great impact on business performance. A positive corporate culture can bring great benefits to an enterprise and make it a leader in the industry. A negative corporate culture has a negative impact on performance and limits the adoption of needed strategies. Therefore, through the reform of the original corporate culture, to shape a better corporate culture, so as to improve corporate performance. Li jun (2002) believed that the construction of enterprise value system (cultural performance) was the core pillar to improve enterprise performance. In the research of excellent enterprises at home and abroad, no matter the enterprise belongs to service industry or manufacturing industry, every spirit or idea guides the enterprise to take every activity. This spirit is the value system contained in the enterprise. These value systems embody the enterprise's goal and are an important part of enterprise culture. It gives everyone in the enterprise the direction and motivation to act and reveals the vision of the enterprise in the future. These value systems should reflect the goals of the enterprise and be as descriptive and quantifiable as possible. Profit should not be the ultimate goal of the enterprise, but should be reflected as a byproduct of the pursuit of other goals.

\section{CONCLUSION}

Non-financial evaluation indicator plays an important role and significance in China's enterprise performance evaluation indicator system. It can dynamically evaluate the process of enterprise business operation and timely solve existing problems. Based on the whole enterprise, it emphasizes the overall performance of the enterprise. More importantly, the non-financial evaluation indicator can promote the sustainable development of the enterprise through the evaluation of the enterprise's innovation ability and value creation ability. However, the non-financial evaluation indicator still has some limitations. Therefore, we must try our best to avoid weaknesses in practice and use non-financial evaluation indicators reasonably and effectively. At the same time, non-financial indicators should be combined with financial evaluation indicators to make up for and correct their deficiencies and weak links in time, so that the overall comprehensive performance evaluation of enterprises can be effectively reflected, so as to effectively improve operating efficiency and development capacity.

\section{REFERENCES}

[1] Wu Bingjie. Research on the correlation between non-financial driving indicators and corporate value [D]. Beijing: Chinese Academy of Fiscal Sciences, 2019

[2] Tong Mengjie. Capitalization of intangible assets, performance evaluation of non-financial indicators and corporate financial performance $[\mathrm{J}]$. Communication of Finance and Accounting,2020 (18)

[3] Wang Huabing, Liu Fang. Research on Collaborative Innovation Mechanism of Non-financial Indicators and Corporate Segment Strategy [J]. Friends of Accounting, 2018 (15)

[4] Wang Chunhui. Research on the Impact of University's Non-financial Indicators on Financial Indicators_-Based on the Perspective of University Function Orientation [J]. Friends of Accounting, 2016 (23)

[5] Lissa. The Strategic Transmission of Financial and Non-Financial Indicators in Performance Management__ Taking Japanese Chain Store Organizations as Objects [J]. Journal of Huaihai Institute of Technology (Humanities and Social Sciences Edition), 2016 (10)

[6] Zeng Deming, Chen Liyong. A Summary of the Research on the Relationship between Stakeholder Governance and Firm Performance [J]. Economics dynamics, 2003(3)

[7] Li jun. Analysis of the Ways to Effectively Improve Enterprise Performance [J]. Business research,2002(3) 